\title{
Upregulation of circadian gene 'hClock' contribution to metastasis of colorectal cancer
}

\author{
YAPING WANG ${ }^{1}$, NING SUN $^{2}$, CHAO LU $^{2}$, YIBING BEI $^{1}$, RUIZHE QIAN $^{2}$ and LUCHUN HUA ${ }^{1}$ \\ ${ }^{1}$ Department of Surgery, Huashan Hospital, Fudan University, Shanghai 200040; ${ }^{2}$ Department of Physiology \\ and Pathophysiology, Shanghai Medical College, Fudan University, Shanghai 200032, P.R. China
}

Received February 20,2017; Accepted April 19, 2017

DOI: 10.3892/ijo.2017.3987

\begin{abstract}
Recent studies have shown that disruption of the circadian rhythm was one of the endogenous factors contributing to tumorigenesis of various human malignancies, including colorectal cancer (CRC). However, the roles of circadian genes in the development of CRC are still unexplored. In this study, we investigated the expression pattern and the underlying mechanism of human Clock gene (hClock) in CRC progression. Multiple methods such as qRT-PCR, immunohistochemistry, and western blotting were performed to evaluate the expression pattern of the gene hClock, as well as to observe the changes of angiogenesis-related proteins and EMT-related proteins. Transwell cell migration assays and an animal tumor metastasis model were used to examine the impact of hClock on the metastatic ability of CRC cells in vitro and in vivo. Our results showed that the expression level of hClock significantly increased in human CRC tissues, which strongly associated with late TNM stage and positive lymph node metastasis. Moreover, a higher level of hClock expression was found in $\mathrm{CRC}$ cell lines with a higher metastatic potential. Furthermore, ectopic expression of hClock promoted the migration of SW480 CRC cells, while knockdown of hClock inhibited the tumor metastasis of SW620 CRC cells, and targeting hClock by shRNA effectively suppressed the metastatic ability of SW620 CRC cells in nude mice. Finally, we found that overexpression of hClock enhanced the expression of angiogenesis-related genes such as HIF- $1 \alpha$, ARNT and VEGF, and promoted epithelial-mesenchymal (-like) transition (EMT) in CRC cells, both of which are considered to be critical for tumor progression. These findings suggest that upregulation of the circadian gene hClock plays an important role in metastasis of colorectal cancer.
\end{abstract}

Correspondence to: Professor Luchun Hua, Department of Surgery, Huashan Hospital, Fudan University, 12 Wulumuqi Zhong Road, Shanghai 200040, P.R. China

E-mail: drhua@126.com

Key words: circadian, human Clock gene, colorectal cancer metastasis

\section{Introduction}

Colorectal cancer (CRC) is one of the commonest malignancies and also the leading cause of cancer-related deaths worldwide. The incidence rates of CRC show an increased trend in the world, including China $(1,2)$. The majority of the CRC patients developed lymph node metastasis, even liver, lung, and peritoneum peritoneal metastasis leading to high rates of death (3). Consequently, much work remains to be done to fully understand the molecular mechanisms of the development of CRC, and the importance of markers that promote the progression of CRC has been emphasized, as they may function as therapeutic targets (4).

Recently, circadian disruption has become the main stay in research field of oncology as it affects the genesis, development and treatment of tumors (5-7). Growing evidence indicates that disruption of circadian rhythms by a set of clock genes and proteins significantly increases the incidence of human CRC. For example, previous studies have reported higher incidence rates of colorectal, breast, and endometrial cancers which existed in shift workers with presumed circadian disruption (8). On the other hand, many clinical trials have reported strong evidence on the beneficial effects of chronotherapy, which refer to the delivery of chemotherapy according to the circadian rhythms $(9,10)$. These studies showed that chronotherapy significantly increased the tolerance to high doses of chemotherapeutic drugs, improved clinical response and prolonged survival in patients with metastatic CRC (11-13). We aimed to clarify who plays the key role in circadian disorder and how it affects CRC progression.

Various living organisms exhibit behavioral and physiological circadian rhythms, allowing them to adapt to daily light and dark cycle (14). The molecular mechanism of circadian oscillations includes eight core circadian genes $(15,16)$ : Period1 (Per1), Period2 (Per2), Period3 (Per3), Clock, Bmal1, Casein Kinase I $\varepsilon$ (CKIE), Cryptochromel (Cryl) and Cryptochrome2 (Cry2). Among them, the gene Clock is an integral and core component of the circadian pacemaking system $(17,18)$.

The current study also investigated the role of the core circadian genes involved in the tumorigenesis of CRC. For example, in the CRC tumor tissues of patients, expression levels of Per1, Per2, Per3 were lower compared to their 
matched healthy mucosa (19-21), and a low expression of the Perl gene was correlated with liver metastasis (22). Moreover, Cryl overexpression in CRC tissues was correlated with tumor progression and poor prognosis (23), and genetic variants in the gene hClock had a significant effect on the risk of death of CRC patients (24). Lu et al, on 250 advanced rectal cancer patients, reported that the effect of neoadjuvant chemotherapy was correlated with expression of the gene Clock. They have further put forward that circadian genes acted as potential biomarkers for predicting the beneficial effects of neoadjuvant chemoradiation therapy in patients with rectal cancer (25).

Above all, since the hClock gene is the core component of the circadian gene family, we assume that disruption of this gene expression may play an important role in the incidence of $\mathrm{CRC}$, especially in the tumor invasion and metastasis. However, there are few studies supporting the variations of hClock expression in the CRC progression, and the inclusion of hClock working mechanism in the invasion and metastasis of CRC still needs investigation.

In our previous studies, we found that the circadian gene human Clock (hClock) was highly expressed in the CRC tissues, and associated with late TNM stage and positive lymph node metastasis (26). Our previous studies also demonstrated the inhibition of tumor cell apoptosis by hClock in vitro and in vivo, while hClock silencing reversed this effect (27). Hence, in this study, we further explored the role and mechanism of hClock participation in CRC progression in vitro and in vivo.

\section{Materials and methods}

Ethics statement. The study was approved by the institutional review board of Huashan Hospital Affiliated to Fudan University (HIRB), Shanghai, China. All patients provided written informed consent. All in vivo experiments in the study protocol were strictly in accordance with the National Institutes of Health Guide for Care and Use of Laboratory Animals and were approved by the Animal Care and Use Committee of Shanghai Medical College of Fudan University, Shanghai, China.

CRC specimens and cell lines. CRC specimens including adjacent non-tumor colorectal tissues were obtained from patients who have undergone radical surgery for CRC in the Huashan Hospital, Shanghai, China. None of the patients received chemotherapy or radiotherapy before operation. The samples were surgically obtained at the following time points: 23 cases between 10:00 and 12:00, 8 cases between 12:00 and 14:00, 3 cases between 14:00 and 16:00, 3 cases between 16:00 and 18:00, and 1 case at 22:00.

Four human CRC cell lines SW480, SW620, HT29, LoVo, and human embryonic kidney cell line 293T were cultured in Dulbecco's modified Eagle's medium (DMEM) with $10 \%$ (v/v) newborn calf serum, $100 \mathrm{U} / \mathrm{ml}$ penicillin and $100 \mathrm{ng} / \mathrm{ml}$ streptomycin at $37^{\circ} \mathrm{C}$ in $5 \% \mathrm{CO}_{2}$. All cell lines were obtained from the Cell Bank of the Chinese Academy of Sciences, Shanghai, China.

Quantitative reverse transcription-PCR, immunohistochemistry and evaluation of staining. Detailed materials and methods performed were according to our previous study (28).
Table I. Relationship between increased expression of hClock protein and clinicopathological features.

\begin{tabular}{lccc}
\hline $\begin{array}{l}\text { Clinicopathological } \\
\text { features }\end{array}$ & $\begin{array}{c}\text { Total } \\
\text { cases } \\
\mathrm{N}\end{array}$ & $\begin{array}{c}\text { Increased hClock } \\
\text { expression in tumor } \\
\text { n (\%) }\end{array}$ & P-value \\
\hline Sex & & & NS \\
Male & 18 & $9(50.0 \%)$ & \\
Female & 20 & $10(50.0 \%)$ & NS \\
Age, yrs. & & & \\
$\leq 50$ & 8 & $5(62.5 \%)$ & NS \\
$>50$ & 30 & $14(46.7 \%)$ & \\
Tumor site & & & 0.003 \\
Colon & 21 & $11(52.4 \%)$ & \\
Rectum & 17 & $8(47.1 \%)$ & \\
Lymph nodes spread & & & \\
Negative & 21 & $6(28.6 \%)$ & \\
Positive & 17 & $13(76.5 \%)$ & \\
TNM stage & & $6(28.6 \%)$ & \\
I+II & 21 & $13(76.5 \%)$ & \\
III+IV & 17 & & \\
\hline
\end{tabular}

NS, not significant.

Western blotting. Total cellular proteins were extracted and separated in SDS-PAGE gel, and western blot analysis was performed according to the standard procedures. GAPDH was used as a loading control on the same membrane. The antibodies used included anti-hClock, anti-VEGF (Abcam, Cambridge, UK), anti-GAPDH, anti-HIF-1 $\alpha$, anti-ARNT, anti-E-cadherin, anti-N-cadherin, anti-vimentin, and antifibronectin (Cell Signaling Technology, Danvers, MA, USA). Band densitometry was performed using Scion Image software (Scion Corp., Fredrick, MD, USA).

Plasmid construction. Full-length cDNA of hClock (Genbank accession number: NM_004898) was amplified and inserted into pcDNA3-Flag vector (Invitrogen, Carlsbad, CA, USA) to generate pcDNA3-Flag-hClock expression plasmid. The cDNA fragment of Flag-hClock was inserted into the pGV186 retroviral vector to generate pGV186-Flag-hClock. All the constructs were confirmed by DNA sequencing.

Construction of lentiviral-delivered hClock shRNA. Detailed materials and methods used in this section were performed according to our previous work (27).

Infection of target cells. Retroviruses carrying the hClock or hClockRNAi were generated by cotransfection of recombinant pGV186 or pGV113 plasmids, respectively with pHelper plasmid into 293T cells using Lipofectamine 2000 (Invitrogen). After $48 \mathrm{~h}$ of incubation, the culture medium containing recombinant virus was harvested and purified by a $0.45 \mu \mathrm{m}$ filter. Target cells were seeded $\left(5 \times 10^{5} /\right.$ well) into 6 -well plates 


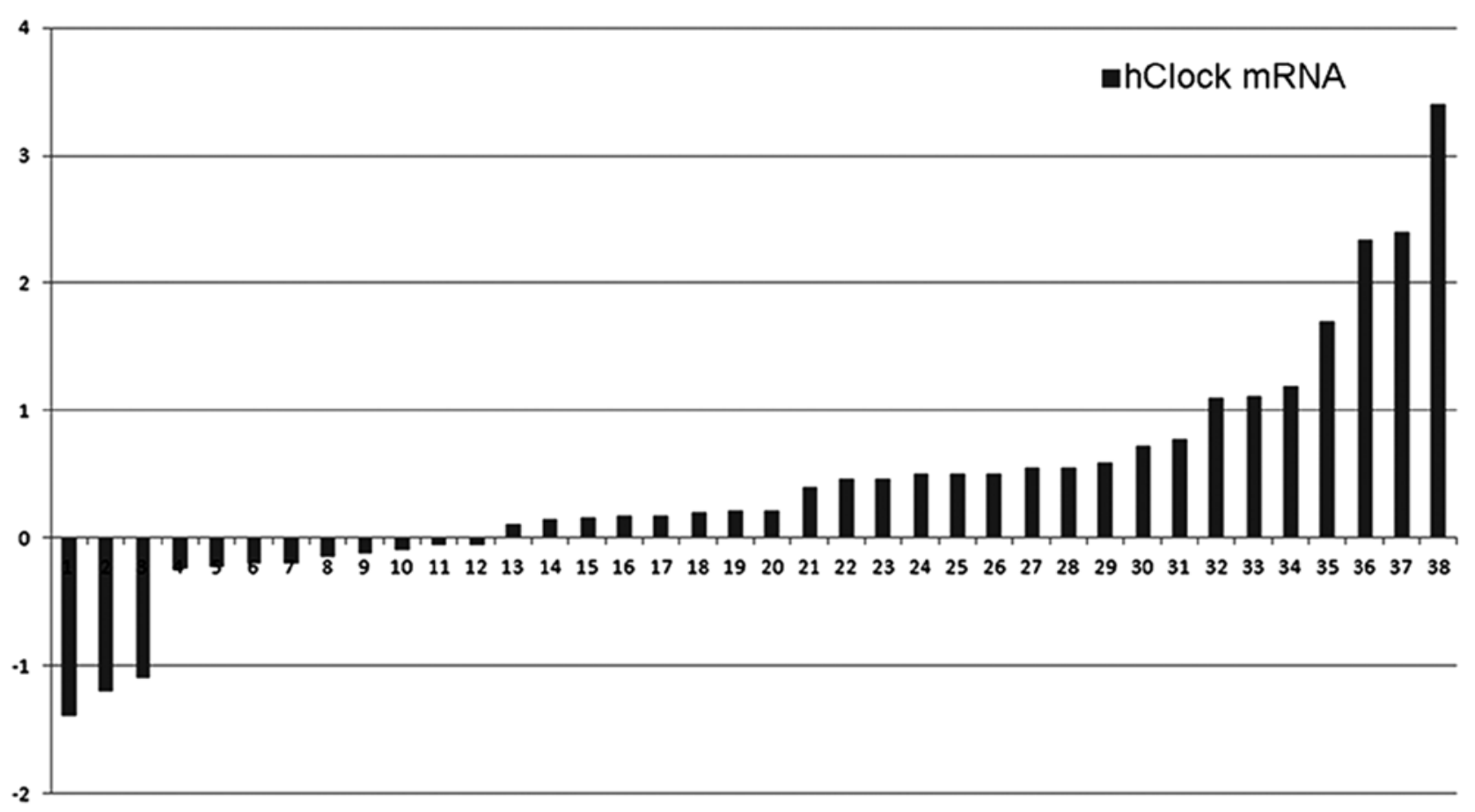

Figure 1. Determination of $h$ Clock mRNA by real-time PCR. $\Delta \mathrm{Ct}(\mathrm{N})$ : Ct value of GAPDH was subtracted from Ct value of $h$ Clock of non-cancerous tissues. $\Delta \mathrm{Ct}(\mathrm{T})$ : $\mathrm{Ct}$ value of GAPDH was subtracted from $\mathrm{Ct}$ value of $h$ Clock of CRC. Bar value $[\Delta \mathrm{Ct}(\mathrm{N})-\Delta \mathrm{Ct}(\mathrm{T})]$ represents the difference of $h C l o c k$ mRNA between non-cancerous tissues and paired CRC tissues. Each bar represents one sample each.

and incubated with recombinant virus was supplemented with $5 \mu \mathrm{g} / \mathrm{ml}$ polybrene for a spin infection procedure.

Cell migration assay. The migration ability of the cells was measured in transwell chambers ( $8 \mu \mathrm{m}$ pore; BD Biosciences). The bottom chamber was filled with $600 \mu \mathrm{l}$ of DMEM containing $10 \%$ FBS. For the migration assay, tumor cells $\left(5 \times 10^{4}\right.$ cells in a total volume of $\left.100 \mu \mathrm{l}\right)$ were placed in the upper chamber and incubated at $37^{\circ} \mathrm{C}$ in $5 \% \quad \mathrm{CO}_{2}$ under humidified air conditions. After $24 \mathrm{~h}$ of culture, non-migrating cells on the upper surface of the membrane were removed, and cells that migrated to the underside of the polycarbonate membrane were fixed with ethanol and stained with $1 \%$ crystal violet for $10 \mathrm{~min}$. The number of migrating cells was then determined from 5 independent microscopic fields. The mean of triplicate assays for each experimental condition was used for the analysis.

Lung metastatic tumor experiment in nude mice. Female, 5-6 weeks old, Balb/c nude mice were obtained from Shanghai Experimental Animal Center and provided with standard laboratory chow and tap water ad libitum under special pathogen free (SPF) conditions in the department of laboratory animal science of Shanghai Medical College of Fudan University. The mice were then separated into 2 groups randomly and were injected with SW620-hClock-shRNA 2\# or SW620-shRNAcontrol infected cells via the caudal veins (10 mice/group, $1 \times 10^{6}$ cells/mouse). Six weeks after injection, all mice were euthanatized by cervical dislocation and their lungs were sectioned and stained with hematoxylin and eosin (H\&E), and then observed by light microscopy for the formation of lung metastasis. All the animal studies were conducted in accor- dance with 'Animal Research: Reporting In vivo Experiments' (ARRIVE) guidelines and the guidelines of Institutional Animal Care and Use Committee. All the mice were treated humanely throughout the experimental period.

Statistical analysis. The data are presented as mean \pm SD unless stated otherwise. A paired t-test was used to test for the differences in hClock expression between matched tumor and benign mucosa. $\chi^{2}$-test or Fisher's exact test was performed to analyze the correlations of hClock protein levels with clinical and pathologic parameters. The statistical significance of the in vitro and in vivo studies was analyzed using Student's t-test. All P-values were two-sided, and P-values of $<0.05$ were considered to be statistically significant. All statistical analyses were conducted using SPSS software package, version 19.0 (SPSS Inc., Chicago, IL, USA).

\section{Results}

Analysis of hClock expression in human CRC specimens and cell lines. In our previous studies, we found that hClock was highly expressed in CRC tissues when compared with the peritumoral tissues in CRC patients, and strongly associated with late TNM stage and positive lymph node metastasis (26). Similar results can be found in the present study since another additional 8 cases were added to the current study and were further analyzed.

We analyzed hClock mRNA levels in 38 pairs of human CRC samples, including primary CRC tissues and paired normal colorectal tissues in this study. Significantly increased expression of $h$ Clock mRNA was observed in 26 of $38(68.4 \%)$ CRCs compared with the paired normal colorectal 
A

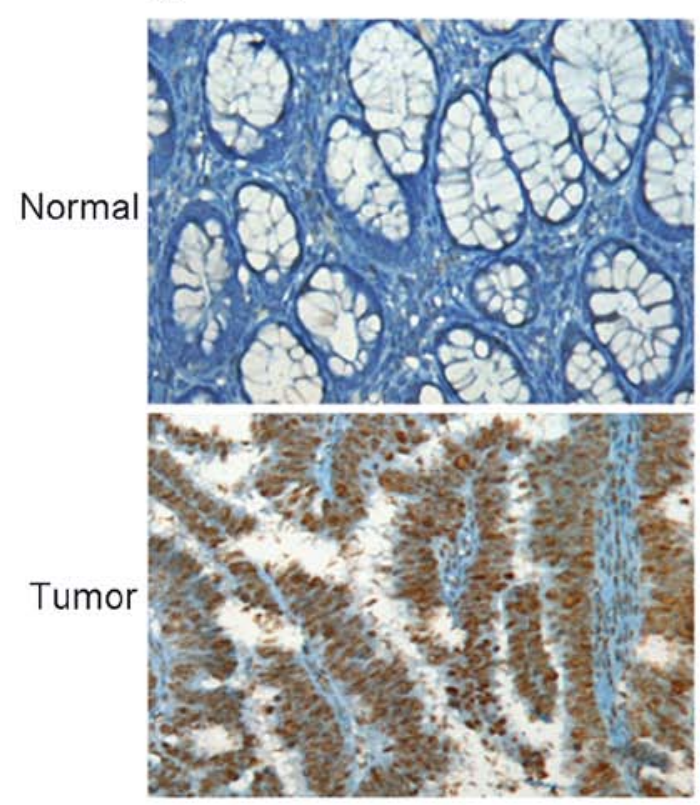

B

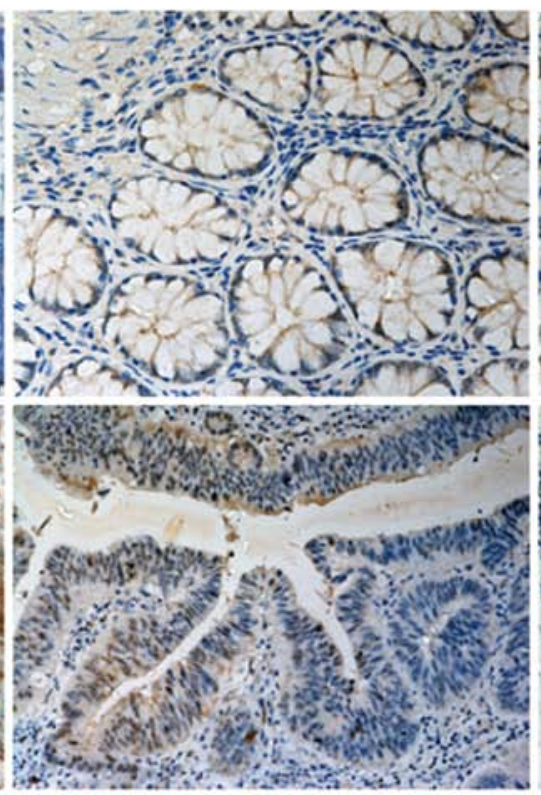

C

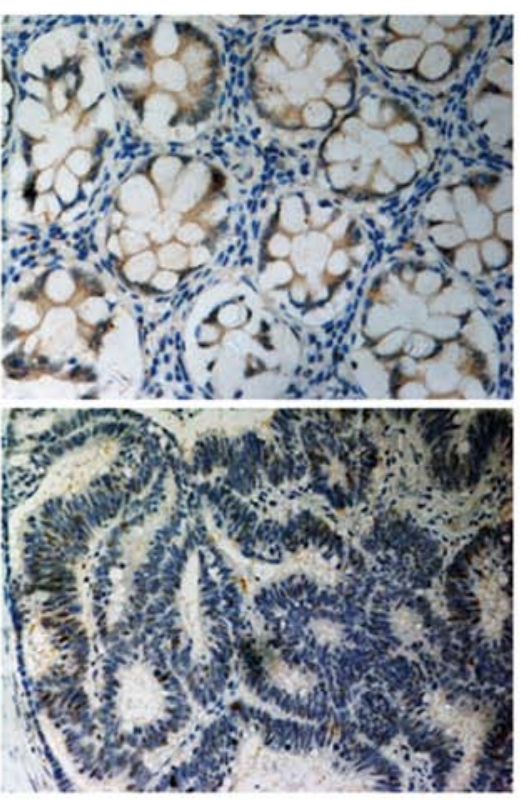

Figure 2. Typical expression patterns of $h$ Clock in CRC tissue samples. (A) Pattern type I, immunohistochemical staining for $h C l o c k$ was much stronger in cancerous cells than in the paired non-cancerous cells (x200). (B) Pattern type II, similar expression of $h$ Clock protein was found in the CRC cells and the paired non-cancerous cells (x200). (C) Pattern type III, staining for hClock was stronger in the non-cancerous cells than in the paired cancerous cells (x200).
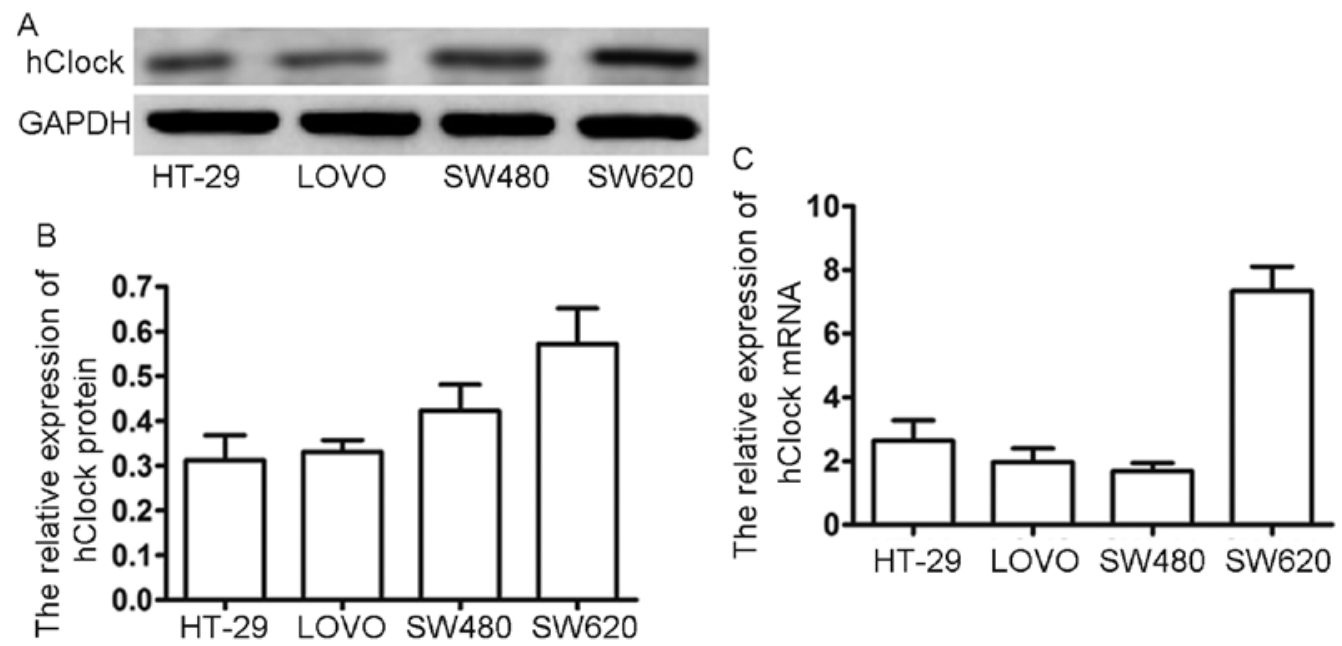

Figure 3. Relative expression levels of $h$ Clock in various CRC cell lines. (A) Relative $h$ Clock protein levels in different cell lines by western blot analysis. (B) Densitometric quantification of data was expressed as the intensity ratio of objective gene to GAPDH. (C) Relative $h C l o c k$ mRNA levels in different cell lines by qRT-PCR analysis.

tissues $(\mathrm{P}<0.01)$. Relative expression levels of hClock in the CRC compared with the non-cancerous components were 2.75:1 (Fig. 1).

hClock expression at the protein level was further examined in these 38 pairs of CRC tissues by immunohistochemical staining. hClock protein was predominantly detected in the nuclei of tumor cells. In all, there were three different $h$ Clock protein expression patterns: type I $(50.0 \%, 19 / 38)$ : staining for $h$ Clock was much stronger in the cancerous cells than in the paired non-cancerous cells (Fig. 2A); type II (44.7\%, 17/38): there were no visually significant differences between the staining of cancerous and non-cancerous cells (Fig. 2B), although subtle differences in the hClock protein expression could not be excluded; type III $(5.3 \%, 2 / 38)$ : staining of
hClock was stronger in non-cancerous cells than in the paired cancerous cells (Fig. 2C). Furthermore, 26 cases showed upregulated expression of hClock mRNA in the CRC tissues, 18 specimens demonstrated a higher expression of $h \mathrm{Clock}$ protein. Thus, the expression of hClock protein and mRNA revealed a positive correlation $(\mathrm{P}<0.01)$.

We next examined the possible intrinsic relationship between $h$ Clock expression and clinicopathological features. As shown in Table I, hClock upregulation did not correlate with sex, age, and tumor sites, but increased expression of hClock was strongly associated with positive lymph node metastasis $(\mathrm{P}=0.003)$ and advanced TNM staging $(\mathrm{P}=0.003)$. These results suggested that higher $h$ Clock expression was associated with the degree of malignancy for CRC. 
A
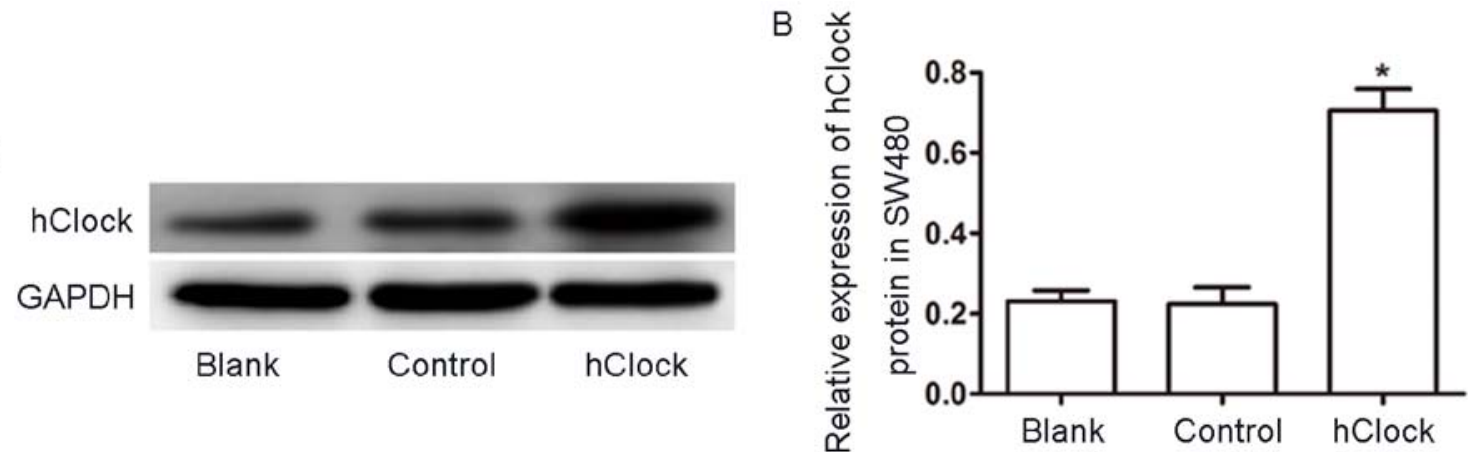

C

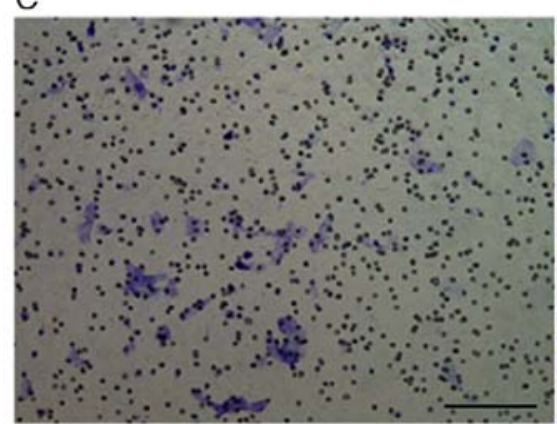

Control

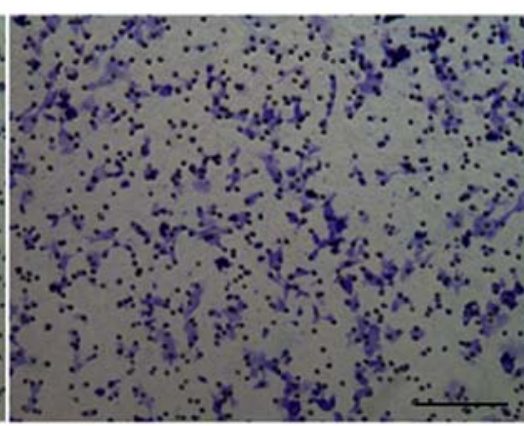

hClock

$200 \mu \mathrm{m}$

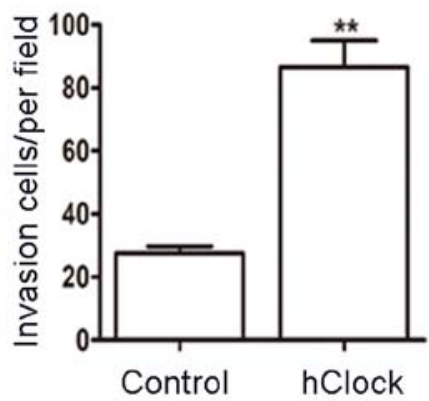

Figure 4. Stable overexpression of hClock in CRC cell line SW480 increased cell proliferation and migration. (A) Western blot analysis indicated that the $h$ Clock gene was stably overexpressed in SW480 cells by infecting with pGV186-hClock lentiviruses. (B) Densitometric quantification of data was expressed as the intensity ratio of $h$ Clock to GAPDH (as an internal reference). $h C l o c k$ protein expression was significantly upregulated in pGV186-hClock transducted SW480 cells compared to that in control lentiviruses-transduced SW480 cells and untreated cells $\left({ }^{*} \mathrm{P}<0.05\right)$. (C) Transwell matrigel invasion assay showed that the number of invasive cells were increased significantly in the $h C l o c k$ overexpressed group when compared with those in the control group (x200, ${ }^{* *} \mathrm{P}<0.01$ ).

To validate our findings from the clinical CRC samples, we examined cellular hClock expression in four different CRC cell lines (HT-29, LoVo, SW480 and SW620). Results of $h$ Clock expression in the four CRC cell lines are shown in Fig. 1. Noteworthy, hClock was highly expressed in SW620 both at the mRNA and protein level (Fig. 3A and B). Since SW620 has the highest potential of metastasis among these CRC cell lines (28), our result again suggested that $h C l o c k$ overexpression significantly correlated with CRC metastasis.

$h$ Clock promotes migration of CRC cells. To evaluate the role of $h$ Clock in CRC, we next tested whether overexpression of $h$ Clock promoted the migration of human CRC cells. We used the CRC cell line SW480 which relatively expressed a lower level of hClock. hClock was overexpressed in SW480 cells when infected with pGV186-hClock lentiviruses (Fig. 4A and B). Transwell migration assay showed that overexpression of $h$ Clock enhanced the migration ability of SW480 CRC cells (Fig. 4C, $\mathrm{P}<0.01)$. These findings suggested that $h$ Clock plays an important role in the CRC cell migration.

To further investigate the relationship between $h$ Clock upregulation and CRC progression, endogeneous hClock expression in the CRC cell line SW620 was knocked down and cell migration was examined. Transfection of SW620 cells with $h$ Clock hRNA significantly inhibited the $h$ Clock expression (Fig. 5A and B). To further examine whether the targeted knockdown of $h$ Clock in SW620 cells affected their migration, we performed an in vitro Transwell migration assay. The result showed that the number of hClock-shRNA cells migrated through the filter decreased markedly by contrast to the control group (Fig. $5 \mathrm{C}, \mathrm{P}<0.01$ ). These results together indicated that $h C l o c k$ was critical for CRC cell proliferation and migration in vitro.

To further explore the role of $h$ Clock in CRC metastasis in vivo, SW620 CRC tumor cells transfected with hClockshRNA or with control hRNA were injected into the nude mice via the caudal vein (10 mice/group, $1 \times 10^{6}$ cells/mouse). Six weeks after injection, none of the mice had died or behaved abnormally, and they were euthanatized. Their lungs were sectioned and stained with $\mathrm{H} \& \mathrm{E}$, and then observed by light microscopy. Notably, in contrast to the control group, a significant reduction in the number of metastatic nodules in the lungs of nude mice injected with hClock-shRNA-transfected SW620 cells was observed (Fig. 5D). These results showed that targeting $h$ Clock by shRNA effectively suppressed the metastatic ability of SW620 CRC cells in nude mice and suggested that $h$ Clock knockdown exerted a strong antitumor effect in vivo.

Taken together, both our in vitro and in vivo experiments provided significant evidence that $h C l o c k$ was strongly associated with CRC metastasis.

Overexpression of hClock promotes epithelial-mesenchymal (-like) transition (EMT) in CRC cells and upregulated the expression of tumor angiogenesis-related genes HIF-1 $\alpha$, $A R N T$ and VEGF. It has been previously reported that EMT was the driving force of invasion in the epithelial cells $(29,30)$. In recent years, changes in the level of expression of different cadherins, so-called cadherin switches 
A

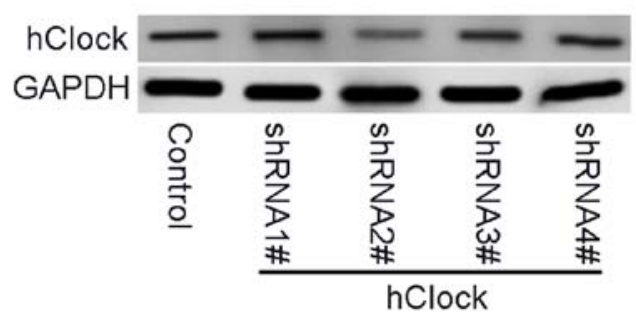

B
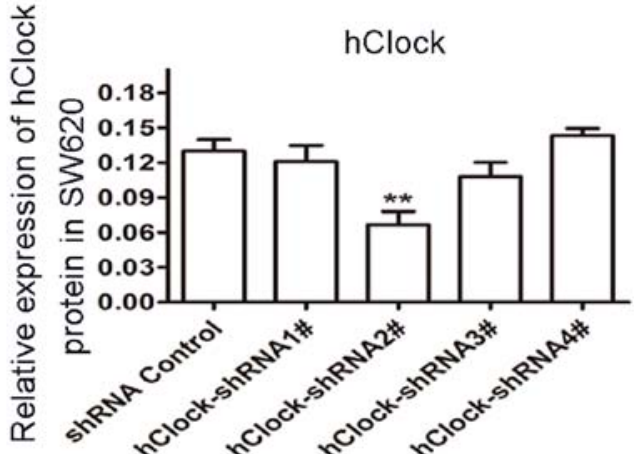

C

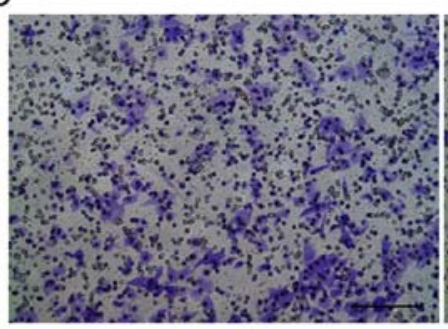

shRNA control

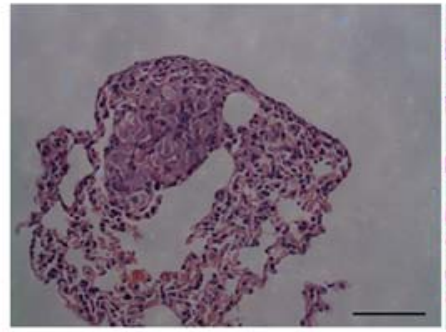

shRNA control

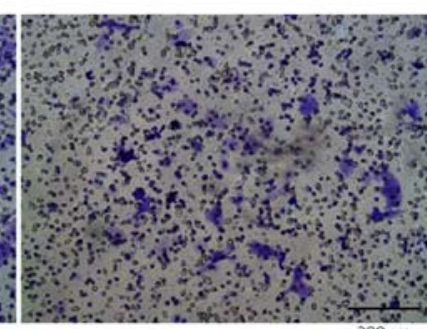

hClock-shRNA 2\#

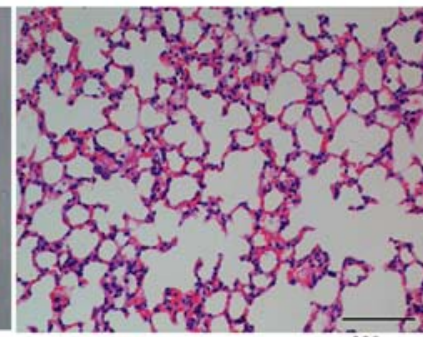

hClock-shRNA 2\#
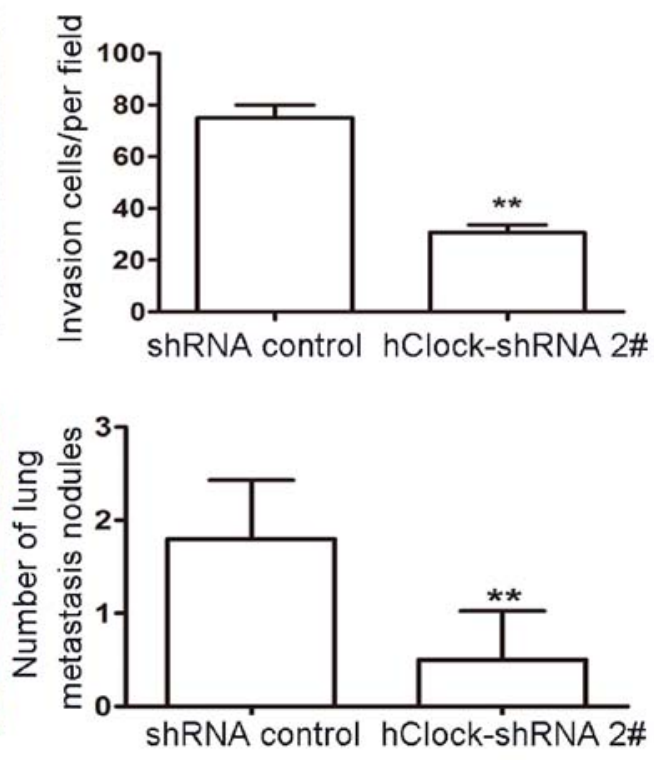

Figure 5. shRNA-mediated knockdown of hClock expression in SW620 CRC cells leads to the inhibition of cell proliferation and tumor metastasis in vitro and in vivo. (A) Western blot analysis of $h$ Clock in shRNA-downregulated $h$ Clock clones (hClock shRNA1\#-4\#) and vector clones (control shRNA) in SW620 cells. (B) Densitometric quantification of data were expressed as the intensity ratio of $h C l o c k$ to GAPDH (as an internal reference), and Clone $2 \#$, which showed significant downregulation of $h$ Clock protein expression compared with the control vector-transduced clones (inhibition efficiency: $50.4 \%$, $\left.{ }^{*} \mathrm{P}<0.05\right)$, which was chosen for further analysis. (C) Transwell migration assay was performed to measure the migration of SW620 CRC cells, crystal violet staining of the lower surface filters which showed that cells were able to migrate through the filter (x200). Average number of cells that were migrated through the filter was counted. The cells of interfering group were significantly less able to migrate than the control group ( $\left.{ }^{* *} \mathrm{P}<0.01\right)$. (D) The representative graph of metastatic nodules, in the lungs of nude mice injected with SW620 cells transduced with control shRNA. In contrast, the representative graph of the lung without metastatic nodules of nude mice injected with hClock-shRNASW620 cells [x200, by hematoxylin and eosin (H\&E)]. The average number of lung tumors was counted. The number of metastatic nodules of interfering group was less than that of the control group $\left({ }^{*} \mathrm{P}<0.05\right)$.

have been increasingly used to monitor EMT. The cadherin switch from E-cadherin to $\mathrm{N}$-cadherin, which is expressed in mesenchymal cells, fibroblasts, cancer cells, and neural tissues has often been used to monitor the EMT progress during the embryonic development and cancer progression (31). Thus, to investigate the mechanism underlying the effects of hClock overexpression on CRC progression, we examined the expression of EMT markers E-cadherin and $\mathrm{N}$-cadherin. As shown in Fig. 6A and B, expression levels of E-cadherin were significantly decreased in SW480 cells which overexpressed hClock compared with those of the vector only-treated SW480 cells $(\mathrm{P}<0.05)$, whereas upregulation of $h$ Clock resulted in a significant increase in $\mathrm{N}$-cadherin expression $(\mathrm{P}<0.05)$. In contrast, silencing of endogenous $h$ Clock expression resulted in a marked decrease of N-cadherin expression compared with that of the shRNAtransfected controls, whereas E-cadherin expression levels were significantly increased $(\mathrm{P}<0.05)$ (Fig. 6A and $\mathrm{C}$ ).
In parallel, increasing evidence demonstrates that circadian genes participate in the angiogenesis and growth of tumors $(32,33)$. Thus, we analyzed the expression of tumor angiogenesis-related genes, such as HIF-1 $\alpha$, ARNT, VEGF in the CRC cell lines with hClock knockdown or overexpression, to see whether ectopic expression of hClock contributes to tumor angiogenesis and malignancy. Western blot analysis indicated that expression of HIF-1 $\alpha$, ARNT and VEGF were decreased in SW620 cells transfected with hClock shRNA, but increased in SW480 cells with hClock overexpression (Fig. 7).

\section{Discussion}

CRC patients frequently develop lymph nodes and hematogenous metastases in the advanced stages. The factors involved in CRC metastasis are largely unknown, however, deregulation of molecular processes and signaling pathways are generally 

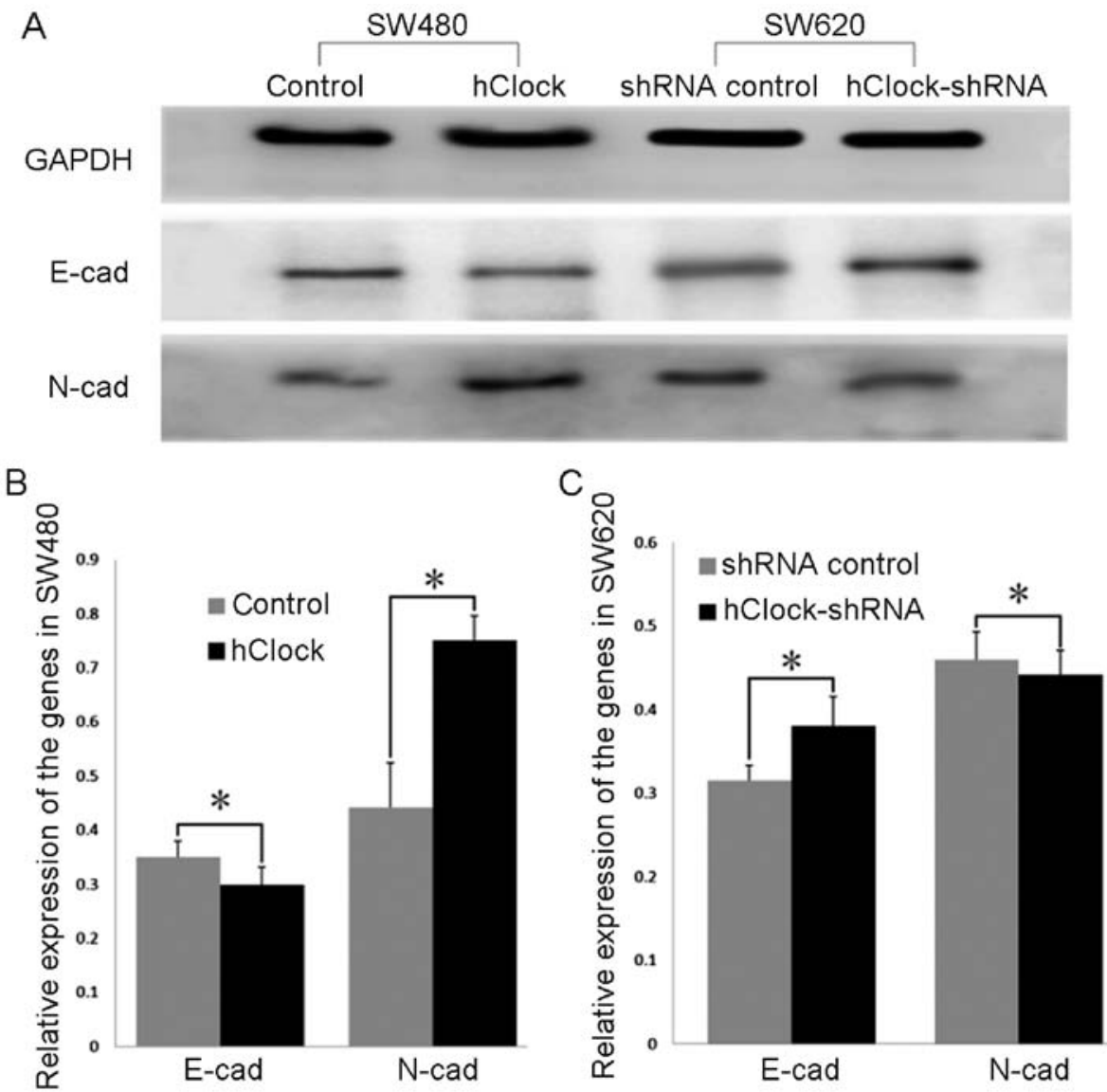

Figure 6. Overexpression of $h$ Clock promoted EMT progression. (A) Western blot analysis of E-cad and N-cad expression levels in SW480 cells with $h C l o c k$ overexpression and in SW620 cells with hClock knockdown. (B and C) Densitometric quantification of data (expressed as the intensity ratio of the objective genes to GAPDH) was calculated by western blot analysis of proteins in (B) SW480 cell overexpression $h C l o c k$ and (C) SW620 cells with downregulated expression of $h$ Clock. Values are presented as mean \pm standard deviation $(\mathrm{n}=3)$. (B) Overexpression of $h C l o c k$ in SW480 cells upregulated the expression of N-cad, while downregulated the expression of E-cad ( $\left.{ }^{*} \mathrm{P}<0.05\right)$. (C) N-cad expression was decreased in SW620 cells which also downregulated $h C l o c k$ expression, and vice versa in E-cad $\left({ }^{*} \mathrm{P}<0.05\right)$.

considered to be the main causes which result in the growth and metastasis of the disease. Consequently, the importance of molecular markers that promote the development of CRC has been emphasized, as they might be the therapeutic targets for the disease (34).

In our present study, we showed that the expression of hClock increased in the CRC tissues significantly. We also showed that overexpression of hClock was strongly associated with lymph node matastasis and late stage of TNM classification in CRC patients. Since these clinicopathological features indicate relatively poor prognosis, overexpression of hClock can be regarded as one of the potential biomarkers for evaluating malignancy of $\mathrm{CRC}$ and requires further investigation in the future. In parallel, we examined the expression of hClock in CRC cell lines, and observed statistically higher expression of hClock in SW620 cells compared to SW480 cells both at mRNA and protein level. SW620 has a higher metastatic potential than SW480 cells (29), this suggests that hClock overexpression has a correlation with CRC metastasis. Overall, these results suggest that disruption of hClock expression is associated with CRC progression.

The role of hClock in CRC progression is still poorly understood. Aiming to investigate this biological question, we first examined in this study the effect of hClock on CRC cell migration through transwell assay. The results revealed that repressed hClock expression by shRNA led to the retardation of cell migration of SW620 CRC cells, while in stable overexpression of hClock in SW480 CRC cells which enhanced cell migration. Experiments of tumor lung metastasis were performed in nude mice which estimated the growthpromoting function of hClock in vivo. Of note, an antitumor effect of hClock RNAi was observed, as lung rectal metastasis was suppressed when hClock expression was knocked down by injecting hClock-shRNA into the nude mice via the caudal vein. Collectively, these results suggest that hClock can be used as a marker to evaluate the progression of CRC, and acts as a strong positive expression of hClock indicating a high risk of lymph node or hematogenous metastasis. Moreover, our data indicated that lentivirus vector-mediated knockdown of hClock in CRC cells significantly inhibited the tumor growth and metastasis both in vitro and in vivo, which indicated a therapeutic potential of hClock shRNA on the treatment of CRC.

Regarding the potential mechanisms underlying the promoting effect of hClock in CRC metastasis, hClock overexpression promoted the EMT progression in CRC cells. We considered that the gene hClock may induce the occurrence of EMT in CRC cells, thus promoting the invasion and metastasis 
A
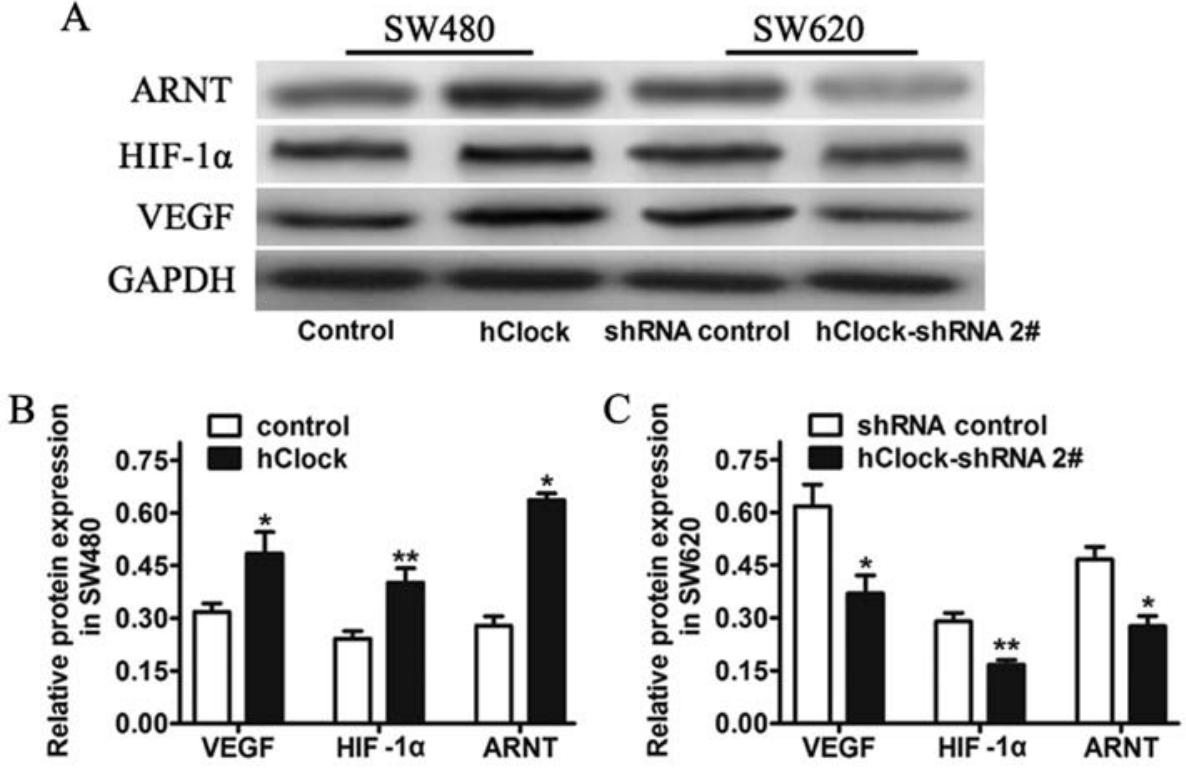

Figure 7. Overexpression of $h$ Clock upregulated the expression of tumor angiogenesis-related genes (HIF-1 $\alpha$, ARNT and VEGF) in CRC cells. (A) Western blot analysis was performed to analyze the expression levels of HIF-1 $\alpha$, ARNT and VEGF which had changed in SW480 cells overexpressing $h C l o c k$ and in SW620 cells transfected with hClock-shRNA. Densitometric quantification of data was expressed as the intensity ratio of objective gene to GAPDH, and are shown in (B and C). Values are presented as mean \pm standard deviation $(n=3)$. (B) Overexpression of $h C l o c k$ in SW480 cells upregulated the expression levels of VEGF, HIF-1 $\alpha$ and ARNT $\left({ }^{*} \mathrm{P}<0.05,{ }^{* *} \mathrm{P}<0.01\right)$. (C) Expression levels of VEGF, HIF-1 $\alpha$ and ARNT were decreased in SW620 cells with downregulation of hClock expression $\left({ }^{*} \mathrm{P}<0.05,{ }^{* *} \mathrm{P}<0.01\right)$.

of CRC. Recently, it was reported that loss of co-repressor circadian gene PER2 under hypoxic conditions upregulated OCT1-mediated EMT gene expression and enhanced tumor malignancy (35). It was also reported that circadian gene Clock contributed to cell proliferation and migration of glioma by upregulating NF- $\kappa \mathrm{B}$ mediated EMT (36). However, to date the role of hClock in the regulation of EMT to promote CRC progression has not been well studied. In combination with the findings of this study, we are interested in research of cytoskeletal protein, transcription factor, adherence protein, or microRNAs which may mediate this pathway. Further efforts may be needed to clarify these issues in our future research.

In 2015, Jensen reviewed recent findings on the role of circadian rhythms and hypoxia in cancer and metastasis, and concluded that circadian rhythms and hypoxia are involved in tumor metastasis at all levels from pathological deregulation of the cells to the tissues and the whole organism. Pathological tumor blood vessels caused hypoxia and disruption in circadian rhythmicity, which in turn commuted to tumor metastasis (37). According to our previous results, expression of hClock was associated with the overexpression of HIF-1 $\alpha$, ARNT and VEGF in CRC specimens (26). We hypothesized that these three genes were clock-controlled genes (CCGs), and their function were regulated by hClock. Thus, in the present study, we further analyzed the expression of these angiogenesis- related genes in CRC cell lines. We found that the expression levels of the genes were all repressed after the knockdown of hClock expression, and were increased in cells with hClock overexpression. VEGF is widely known to play a pivotal role in the tumoral angiogenesis and promote the invasion and metastasis of cancer cells (38). This progression is induced by hypoxia along with the major angiogenic transcription factor hypoxia-inducible factor-1 (HIF-1), which consists of HIF-1 $\alpha$ and HIF-1 $\beta$ (aryl hydrocarbon receptor nuclear translocator, ARNT) (39). Since angiogenesis is essential for the development, growth and advancement of solid tumors (40), our findings implicated that aberrant overexpression of hClock may accelerate the angiogenesis pathway in $\mathrm{CRC}$, and may therefore provide a molecular basis for promoting CRC progression.

In conclusion, our study demonstrated that overexpression of circadian gene $h$ Clock played an important role in the CRC progression by promoting EMT and angiogenesis of the CRC cells. Silencing hClock inhibits the metastasis of CRC cells both in vitro and in vivo. Thus, the gene hClock may serve as a new diagnostic marker in CRC with high metastastic potential as well as a potential therapeutic target for CRC therapy.

\section{Acknowledgements}

This work was supported by the National Science Foundation Fostering Talents in Basic Research of China (no. J1210041, RZ.Q.); the National Natural Science Foundation of China (NSFC) (no. 81570771, RZ.Q.).

\section{References}

1. Siegel RL, Miller KD and Jemal A: Cancer statistics, 2015. CA Cancer J Clin 65: 5-29, 2015.

2. Chen W, Zheng R, Baade PD, Zhang S, Zeng H, Bray F, Jemal A, Yu XQ and He J: Cancer statistics in China, 2015. CA Cancer J Clin 66: 115-132, 2016.

3. Muzny DM, Bainbridge MN, Chang K, Dinh HH, Drummond JA, Fowler G, Kovar CL, Lewis LR, Morgan MB, Newsham IF, et al; Cancer Genome Atlas Network: Comprehensive molecular characterization of human colon and rectal cancer. Nature 487: 330-337, 2012. 
4. Fearon ER: Molecular genetics of colorectal cancer. Annu Rev Pathol 6: 479-507, 2011.

5. Fu L and Lee CC: The circadian clock: Pacemaker and tumour suppressor. Nat Rev Cancer 3: 350-361, 2003.

6. Takahashi JS, Hong HK, Ko CH and McDearmon EL: The genetics of mammalian circadian order and disorder: Implications for physiology and disease. Nat Rev Genet 9: 764-775, 2008.

7. Sahar S and Sassone-Corsi P: Metabolism and cancer: The circadian clock connection. Nat Rev Cancer 9: 886-896, 2009.

8. Straif K, Baan R, Grosse Y, Secretan B, El Ghissassi F, Bouvard V, Altieri A, Benbrahim-Tallaa L and Cogliano V; WHO International Agency For Research on Cancer Monograph Working Group: Carcinogenicity of shift-work, painting, and fire-fighting. Lancet Oncol 8: 1065-1066, 2007.

9. Innominato PF, Focan C, Gorlia T, Moreau T, Garufi C, Waterhouse J, Giacchetti S, Coudert B, Iacobelli S, Genet D, et al; Chronotherapy Group of the European Organization for Research and Treament of Cancer: Circadian rhythm in rest and activity: A biological correlate of quality of life and a predictor of survival in patients with metastatic colorectal cancer. Cancer Res 69: 4700-4707, 2009.

10. Block KI, Block PB, Fox SR, Birris JS, Feng AY, de la Torre M, Nathan D, Tothy P, Maki AK and Gyllenhaal C: Making circadian cancer therapy practical. Integr Cancer Ther 8: 371-386, 2009

11. Giacchetti S, Perpoint B, Zidani R, Le Bail N, Faggiuolo R, Focan C, Chollet P, Llory JF, Letourneau Y, Coudert B, et al: Phase III multicenter randomized trail of oxaliplatin added to chronomodulated fluorouracil-leucovorin as first-line treatment of metastatic colorectal cancer. J Clin Oncol 18: 136-147, 2000.

12. Giacchetti S: Chronotherapy of colorectal cancer. Chronobiol Int 19: 207-219, 2002

13. Focan C, Kreutz F, Graas M-P, Longrée L, Focan-Henrard D, Demolin G and Moeneclaey N: Phase I - II study to assess the feasibility and activity of the triple combination of 5-fluorouracil/ folinic acid, carboplatin and irinotecan (CPT-11) administered by chronomodulated infusion for the treatment of advanced colorectal cancer. Final report of the BE-1603 study. Pathol Biol (Paris) 61: e27-e31, 2013.

14. Gery $\mathrm{S}$ and Koeffler HP: The role of circadian regulation in cancer. Cold Spring Harb Symp Quant Biol 72: 459-464, 2007.

15. Lee C, Etchegaray JP, Cagampang FR, Loudon AS and Reppert SM: Posttranslational mechanisms regulate the mammalian circadian clock. Cell 107: 855-867, 2001.

16. Reppert SM and Weaver DR: Coordination of circadian timing in mammals. Nature 418: 935-941, 2002.

17. Antoch MP, Song EJ, Chang AM, Vitaterna MH, Zhao Y, Wilsbacher LD, Sangoram AM, King DP, Pinto LH and Takahashi JS: Functional identification of the mouse circadian Clock gene by transgenic BAC rescue. Cell 89: 655-667, 1997.

18. Wu D, Potluri N, Lu J, Kim Y and Rastinejad F: Structura integration in hypoxia-inducible factors. Nature 524: 303-308, 2015.

19. Krugluger W, Brandstaetter A, Kállay E, Schueller J, Krexner E, Kriwanek S, Bonner E and Cross HS: Regulation of genes of the circadian clock in human colon cancer: Reduced period-1 and dihydropyrimidine dehydrogenase transcription correlates in high-grade tumors. Cancer Res 67: 7917-7922, 2007.

20. Mazzoccoli G, Panza A, Valvano MR, Palumbo O, Carella M, Pazienza V, Biscaglia G, Tavano F, Di Sebastiano P, Andriulli A, et al: Clock gene expression levels and relationship with clinical and pathological features in colorectal cancer patients. Chronobiol Int 28: 841-851, 2011.

21. Wang Y, Hua L, Lu C and Chen Z: Expression of circadian clock gene human Period2 (hPer2) in human colorectal carcinoma. World J Surg Oncol 9: 166, 2011.

22. Oshima T, Takenoshita S, Akaike M, Kunisaki C, Fujii S, Nozaki A, Numata K, Shiozawa M, Rino Y, Tanaka K, et al: Expression of circadian genes correlates with liver metastasis and outcomes in colorectal cancer. Oncol Rep 25: 1439-1446, 2011.
23. Yu H, Meng X, Wu J, Pan C, Ying X, Zhou Y, Liu R and Huang W: Cryptochrome 1 overexpression correlates with tumor progression and poor prognosis in patients with colorectal cancer. PLoS One 8: e61679, 2013.

24. Zhou F, He X, Liu H, Zhu Y, Jin T, Chen C, Qu F, Li Y, Bao G, Chen Z, et al: Functional polymorphisms of circadian positive feedback regulation genes and clinical outcome of Chinese patients with resected colorectal cancer. Cancer 118: 937-946, 2012.

25. Lu H, Chu Q, Xie G, Han H, Chen Z, Xu B and Yue Z: Circadian gene expression predicts patient response to neoadjuvant chemoradiation therapy for rectal cancer. Int $J$ Clin Exp Pathol 8: 10985-10994, 2015.

26. Wang L, Chen B, Wang Y, Sun N, Lu C, Qian R and Hua L: hClock gene expression in human colorectal carcinoma. Mol Med Rep 8: 1017-1022, 2013.

27. Wang Y, Qian R, Sun N, Lu C, Chen Z and Hua L: Circadian gene hClock enhances proliferation and inhibits apoptosis of human colorectal carcinoma cells in vitro and in vivo. Mol Med Rep 11: 4204-4210, 2015

28. Kubens BS and Zänker KS: Differences in the migration capacity of primary human colon carcinoma cells (SW480) and their lymph node metastatic derivatives (SW620). Cancer Lett 131: 55-64, 1998

29. Mani SA, Guo W, Liao MJ, Eaton EN, Ayyanan A, Zhou AY, Brooks M, Reinhard F, Zhang CC, Shipitsin M, et al: The epithelial-mesenchymal transition generates cells with properties of stem cells. Cell 133: 704-715, 2008

30. Yang MH, Hsu DS, Wang HW, Wang HJ, Lan HY, Yang WH, Huang $\mathrm{CH}$, Kao SY, Tzeng $\mathrm{CH}$, Tai SK, et al: Bmil is essential in Twist1-induced epithelial-mesenchymal transition. Nat Cell Biol 12: 982-992, 2010.

31. Zeisberg $M$ and Neilson EG: Biomarkers for epithelialmesenchymal transitions. J Clin Invest 119: 1429-1437, 2009.

32. Koyanagi S, Kuramoto $Y$, Nakagawa H, Aramaki H, Ohdo S, Soeda $\mathrm{S}$ and Shimeno H: A molecular mechanism regulating circadian expression of vascular endothelial growth factor in tumor cells. Cancer Res 63: 7277-7283, 2003.

33. Jensen LD and Cao Y: Clock controls angiogenesis. Cell Cycle 12: 405-408, 2013

34. Singh R, Lillard JW Jr and Singh S: Chemokines: Key players in cancer progression and metastasis. Front Biosci (Schol Ed) 3: 1569-1582, 2011.

35. Hwang-Verslues WW, Chang PH, Jeng YM, Kuo WH, Chiang PH, Chang YC, Hsieh TH, Su FY, Lin LC, Abbondante S, et al: Loss of corepressor PER2 under hypoxia up-regulates OCT1mediated EMT gene expression and enhances tumor malignancy. Proc Natl Acad Sci USA 110: 12331-12336, 2013.

36. Li A, Lin X, Tan X, Yin B, Han W, Zhao J, Yuan J, Qiang B and Peng X: Circadian gene Clock contributes to cell proliferation and migration of glioma and is directly regulated by tumorsuppressive miR-124. FEBS Lett 587: 2455-2460, 2013.

37. Jensen LD: The circadian clock and hypoxia in tumor cell de-differentiation and metastasis. Biochim Biophys Acta 1850: $1633-1641,2015$

38. Geva E and Jaffe RB: Role of vascular endothelial growth factor in ovarian physiology and pathology. Fertil Steril 74: 429-438, 2000.

39. Wang GL and Semenza GL: Purification and characterization of hypoxia-inducible factor 1. J Biol Chem 270: 1230-1237, 1995.

40. Folkman J: Tumor angiogenesis. Adv Cancer Res 43: 175-203, 1985. 